\title{
Theory of Mind in Children with Traumatic Brain Injury
}

\author{
Maureen Dennis ${ }^{1,2}$, Nevena Simic ${ }^{1,3}$, H. Gerry Taylor ${ }^{4}$, Erin D. Bigler ${ }^{5,6}$, Kenneth Rubin ${ }^{7}$, \\ Kathryn Vannatta ${ }^{8,9}$, Cynthia A. Gerhardt ${ }^{8,9}$, Terry Stancin ${ }^{4,10}$, Caroline Roncadin ${ }^{11}$, and \\ Keith Owen Yeates 8,9
}

\begin{abstract}
${ }^{1}$ Program in Neuroscience \& Mental Health, The Hospital for Sick Children, Toronto, Ontario ${ }^{2}$ Department of Surgery, University of Toronto, Toronto, Ontario ${ }^{3}$ Department of Psychology, University of Toronto, Toronto, Ontario ${ }^{4}$ Department of Pediatrics, Case Western Reserve University, Rainbow Babies \& Children's Hospital, University Hospitals Case Medical Center, Cleveland, Ohio ${ }^{5}$ Department of Psychology, Brigham Young University, Provo, Utah ${ }^{6}$ Department of Psychiatry, University of Utah, Salt Lake City, Utah ${ }^{7}$ Department of Psychology, University of Maryland, College Park, Maryland ${ }^{8}$ Department of Pediatrics, The Ohio State University, Columbus, Ohio ${ }^{9}$ Center for Biobehavioral Health, The Research Institute at Nationwide Children's Hospital, Columbus, Ohio ${ }^{10}$ Department of Psychiatry, MetroHealth Medical Center, Cleveland, Ohio ${ }^{11}$ Psychology Department, Peel Children's Centre, Mississauga, Ontario
\end{abstract}

\begin{abstract}
Theory of mind (ToM) involves thinking about mental states and intentions to understand what other people know and to predict how they will act. We studied ToM in children with traumatic brain injury (TBI) and age- and gender-matched children with orthopedic injuries (OI), using a new three-frame Jack and Jill cartoon task that measures intentional thinking separate from contingent task demands. In the key ToM trials, which required intentional thinking, Jack switched a black ball from one hat to another of a different color, but Jill did not witness the switch; in the otherwise identical non-ToM trials, the switch was witnessed. Overall accuracy was higher in children with OI than in those with TBI. Children with severe TBI showed a larger decline in accuracy on ToM trials, suggesting a specific deficit in ToM among children with severe TBI. Accuracy was significantly higher on trials following errors than on trials following correct responses, suggesting that all groups monitored performance and responded to errors with increased vigilance. TBI is associated with poorer intentional processing in school-age children and adolescents relative to peers with OI; furthermore, children with TBI are challenged specifically by intentional demands, especially when their injury is severe.
\end{abstract}

\section{Keywords}

Intentionality; Childhood head injury; Glasgow Coma Scale; Social cognition; Social problem solving; Error monitoring

Copyright (C) INS. Published by Cambridge University Press, 2012.

Correspondence and reprint requests to: Maureen Dennis, Department of Psychology, The Hospital for Sick Children, 555 University Avenue, Toronto, ON. Canada, M5G 1X8. maureen.dennis@ sickkids.ca.

We have no conflicts of interest to declare. 


\section{INTRODUCTION}

Theory of mind (ToM), a component of social cognition, involves mindreading, the ability to think about mental states in oneself and others and to use this information to understand what other people know and predict how they will act. The term ToMemphasizes that individuals see themselves and others in terms of mental states that result in (and from) human action (Wellman, Cross, \& Watson, 2001). Successful ToM is presumptive evidence that people inhabit a mental world as well as a world of real situations and events and that the two worlds may sometimes diverge. The paradigmatic developmental ToM task involves false belief (Wimmer \& Perner, 1983), the understanding that people can entertain beliefs that contradict reality and that they will act in accordance with their beliefs (e.g., children will search for a candy under a red cup if they believe it to be hidden there, even when it is actually hidden elsewhere).

ToM ability was first studied in great apes (Premack \& Woodruff, 1978). In humans, basic ToM begins early in childhood, with infants displaying expectations about the actions of others and 18-month-olds showing some understanding of mental states (Meltzoff, 1995; Meltzoff, Gopnik, \& Repacholi, 1999). It undergoes extended development from infancy to middle childhood (Bartsch \& Wellman, 1989; Sodian, Taylor, Harris, \& Perner, 1991) and beyond (Frith \& Frith, 2003; Robinson, 2003). In both adults and children, ToM is a component of flexible social function (Astington, 2003; Filippova \& Astington, 2008), including skills such as faux pas and humor (Stuss, Gallup, \& Alexander, 2001).

Interest in ToM moved from the relatively narrow developmental context in which it was originally studied to behavioral and neurological pathologies of adults (Bibby \& McDonald, 2005) and children. ToM, broadly construed, is impaired in various developmental disorders that are associated with poor social function, including autism (Hill \& Frith, 2003), ADHD (Uekermann et al., 2010), spina bifida (Dennis \& Barnes, 1993), and fetal alcohol spectrum disorder (Greenbaum, Stevens, Nash, Koren, \& Rovet, 2009). Poor ToM has also been reported in acquired brain disorders of childhood, including traumatic brain injury (TBI) (e.g., Martín-Rodríguez \& León-Carrión, 2010). Children with TBI display impairments in social-affective functions, including pragmatic language, the understanding of mental state language, the production of speech acts, the understanding of the social function of emotional expressions, the comprehension of the intentions involved in exerting social influence through sarcastic or empathic communications, and the ability to produce coherent social discourse (Chapman et al., 2004; Dennis, Wilkinson, \& Humphreys, 1998; Dennis \& Barnes, 2000, 2001; Dennis, Purvis, Barnes, Wilkinson, \& Winner, 2001; Yeates et al., 2007).

The increasing interest in ToM created problems of scope and problems of measurement. As the scope of ToM became wider, the term ToM came to be applied to a broad range of constructs, including not only the original cognitive belief-based abilities but also empathic responses to other people's pain (e.g., Hein et al., 2010; Olsson et al., 2007; Singer et al., 2004), humor, and social appropriateness (Shammi \& Stuss, 1999). Because so much was subsumed under the ToM umbrella, its measurement became inconsistent. In some studies, poor ToM is inferred from parent or self ratings (Muscara, Catroppa, Eren, \& Anderson, 2009), which are at least one step removed from the child's actual cognitive performance.

The scope problem has been addressed by partitioning ToM into cognitive ToM (which is concerned with cognitive beliefs and reading the information content of people's minds) and affective ToM (which includes emotions and functions involving affective influence, such as empathy). The cognitive versus affective distinction is supported by new behavioral and neuroimaging evidence (Hein \& Singer, 2008; Shamay-Tsoory \& Aharon-Peretz, 2007). The 
measurement problem continues to be somewhat unresolved however, especially given the dearth of cognitive ToM tasks for school aged children.

In this study, we are concerned, narrowly, with cognitive ToM and how it is affected by childhood TBI. Our primary aim is to study ToM using a new task suitable for school-age children and beyond, in which we isolate individual trials that require intentional thinking (mindreading) and compare them with otherwise identical trials that do not, thereby providing a measure of ToM separate from related or contingent cognitive processes. In comparing children with TBI to age- and gender-matched children with orthopedic injuries (OI), we predict that overall test performance will be lower for those with TBI, and, furthermore, that group differences will be non-existent or small for items that do not require ToM processing but will be larger or significant for items requiring ToM.

A secondary aim concerns performance monitoring, the ability to detect failures in one's own performance and to adjust subsequent action. Performance monitoring is disrupted after childhood TBI (Dennis, Barnes, Donnelly, Wilkinson, \& Humphreys, 1996; Hanten et al., 2004; Ornstein et al., 2009), and is behaviorally and neurologically dissociable from performance level in healthy adults (e.g., Chevrier, Noseworthy, \& Schachar, 2007). Performance monitoring is a meaningful question to ask in the context of a study of ToM and non-ToM items because it provides information, not only about self-monitoring skill, but also about the extent to which children with TBI register ToM errors when they make them and adjust their ongoing performance. We compare accuracy and response times on trials following errors to the same outcomes on trials following correct responses. We predict that children with TBI will be less likely to monitor their performance (i.e., will not show increased accuracy or reaction time after errors).

A final secondary aim is to explore the role of TBI severity as a predictor of performance by a traditional metric, the Glasgow Coma Scale (GCS; Teasdale \& Jennett, 1974), and by a rating scale based on the number of abnormalities reported from the time-of-injury computed tomography (CT) scan. Based on recent findings showing that time-of-injury CT reports discriminate outcomes after mild TBI (Levin et al., 2008), we tentatively predict that CT information will enhance group outcome discrimination above and beyond GCS ratings.

\section{METHOD}

\section{Participants}

Participants included children previously hospitalized for either a TBI or OI who were 8 to 13 years of age and who were injured between 6 and 48 months before testing. All children were injured after 3 years of age, the majority after 4 years of age.

For both TBI and OI groups, we applied the following exclusion criteria: (a) history of more than one serious injury requiring medical treatment; (b) premorbid neurological disorder or mental retardation; (c) any injury resulting from child abuse or assault; (d) a history of severe psychiatric disorder requiring hospitalization before the injury; (e) sensory or motor impairment that prevented valid administration of study measures; (f) primary language other than English; and (g) any medical contraindication to MRI or behavioral study. Children in full-time special education classrooms were excluded (in all but one case), although those with a history of premorbid learning or attention problems were not excluded.

Recruitment occurred in three metropolitan sites: Toronto (Canada), Columbus (US), and Cleveland (US). Among children eligible to participate and approached about the study, 82 (47\%) of those with TBI and 61 (26\%) of those with OI agreed to enroll. The participation 
rate was significantly higher for TBI than OI participants. However, participants and nonparticipants in both groups did not differ in age at injury, age at initial contact about the study, sex, race, or census tract measures of socioeconomic status (i.e., mean family income, percentage of minority heads of household, and percentage of households below the poverty line). Participants and non-participants also did not differ on measures of injury severity (i.e., mean length of stay, median Glasgow Coma Scale score for children with TBI).

The human data included in this manuscript were obtained in compliance with formal ethics review committees at the participating institutions in Columbus, Toronto, and Cleveland. Parent consent and child assent was obtained before testing. All participants were assessed a minimum of 6 months post injury. Three participants did not complete the present portion of the study. Of the remaining 140 children, 79 had sustained a TBI. The TBI group had a lowest Glasgow Coma Scale (GCS; Teasdale \& Jennett, 1974) score of 12 or less after resuscitation, or 13-15 score with positive imaging for brain insult or depressed skull fracture. TBI children were grouped by injury severity: GCS scores 9-15 defined a complicated Mild/Moderate TBI group $(n=56)$ and GCS scores 3-8 defined a Severe TBI group $(n=23)$. The OI group $(n=61)$ consisted of children who sustained fractures not associated with any loss of consciousness or other risks or indications of brain injury (e.g., skull or facial fractures) but that involved hospital admission.

Participant demographics, including sex, race, socioeconomic status (Yeates \& Taylor, 1997), WASI IQ, age at injury, age at time of test, and time since injury, mechanism of injury and day-of-injury CT information are shown in Table 1. The socioeconomic composite index (SCI, Yeates \& Taylor, 1997) was significantly higher for OI than for either TBI group, with the Severe TBI group having the lowest mean SCI. The groups also differed in the distribution of mechanism of injury, with injuries arising from motorized vehicles being most common among the Severe TBI group and those arising from sports and recreational events being most common among the OI group. The group differences in SCI were no longer significant when injury mechanism was taken into account. Therefore, we did not treat SCI as a covariate in data analyses, because the SCI differences appeared to be intrinsic to the injury groups. When a covariate is an attribute of a disorder, or is intrinsic to the condition, it is not meaningful and can be potentially misleading to "adjust" for differences in the covariate (Dennis et al., 2009). Our findings are consistent with epidemiological studies showing that the risk of TBI, particularly those linked to motorized vehicles, is highest for children of lower SCI and minority status (Brown, 2010; Howard, Joseph, \& Natale, 2005; Langlois, Rutland-Brown, \& Thomas, 2005; McKinlay et al., 2010; Parslow et al., 2005; Yates, Williams, Harris, Round, \& Jenkins, 2006).

\section{Measure}

The Jack and Jill Task-Participants were shown sequences of three cartoon frames on a computer screen. Each frame included a character (Jack and/or Jill), two hats (red and blue), and a ball. Frame A of each sequence showed Jack placing a ball above a blue or a red hat, an event witnessed by Jill. Frame B showed Jack dropping the ball further down, either into the same hat or into the hat of a different color (unswitched vs. switched). Frame B was either seen or not seen by Jill (witnessed vs. unwitnessed). Frame C showed Jill "thinking" about either a blue or a red hat that contained the ball that Jack dropped. Participants answered yes (by pressing $\sqrt{ }$ on the keyboard) if Frame $\mathrm{C}$ represented what was now in Jill's mind about the ball's location, and $n o$ (by pressing $\mathrm{X}$ on the keyboard) if it did not represent what Jill was thinking. Sample trials for each of the four conditions defined by the switch and witness type are illustrated in Figure 1.

The task consisted of 4 practice trials and 32 test trials, with the order of test trials randomized within and between participants. Switch and witness trial types were balanced 
so that there were eight trials of each possible combination (i.e., switched/witnessed, switched/unwitnessed, unswitched/witnessed, unswitched/unwitnessed). Intentional ToM is involved in the task because Jill's judgment depends on what she believes about the ball's location, not its actual location: Jill will choose the original (Frame A) hat if she is not witness to a switch. Thus, the key trials assessing ToM are those involving an unwitnessed switch of hat color; children need to appreciate that Jill will think the ball is in the original hat although they know it is actually located in the other. The working memory demands are the same in each item; in the ToM and non-ToM information in Frame B, information about whether the switch was witnessed or unwitnessed must be kept in working memory. While the content of working memory changes ( just as it does in a N-back working memory task involving changing strings of digits or letters), the demands on working memory do not. Participants were not given feedback during the task.

Accuracy and reaction time (RT) for correct responses were coded. A score of 1 was given on trials on which participants correctly identified whether the thought bubble accurately represented what Jill was thinking. A score of zero was given on trials on which they did not correctly identify whether the thought bubble accurately represented Jill's thoughts. In addition, if a participant failed to respond within 5 seconds of the third frame (i.e., before the next trial started), a score of 0 was assigned. "Timed out" responses were excluded from subsequent analysis, as were responses made before $100 \mathrm{~ms}$, which were considered to be anticipatory responses. Three participants (2 TBI, 1 OI) did not complete all 32 test trials, completing 25, 27 and 21 trials respectively. Data were analyzed with and without these incomplete responders; results did not differ when incomplete responders were excluded, so results are presented for all children.

Data were first analyzed for normality using the Shapiro-Wilk test; because accuracy and RT data were not normally distributed, data were submitted to an aligned rank transformation, to facilitate a non-parametric factorial analysis (Wobbrock, Findlater, Gergle, \& Higgins, 2011). Transformed accuracy and RT data were first analyzed using group membership as a between-subjects factor and the two trial types (switched $v S$. unswitched, witnessed $v S$. unwitnessed), as within-subjects factors, in repeated-measures analyses of variance. To investigate ToM, we examined the decline in accuracy on switched/ unwitnessed trials as contrasted with switched/witnessed trials, using single-degree-offreedom planned contrasts that compared the Severe TBI group to the OI group and the Mild/Moderate TBI group to the OI group. The effects of age at injury and age at testing on performance were investigated with bivariate Spearman correlations between each factor and several accuracy measures (overall, ToM, and non-ToM) within each group. To examine performance monitoring, transformed accuracy and RT data were analyzed using group membership and trial type (after correct response vs. after error) as between and within-subjects factors, respectively, in repeated-measures analyses of variance.

We explored whether time-of injury CT reports were related to performance in the TBI group, independent of the GCS score that was the basis of group assignment. Reports from CT scans for each participant were coded independently by authors MD and KY for the presence of various lesion types, with one point assigned for the presence of each abnormality. These included focal injuries (focal intracranial contusion; intraparenchymal, intracerebral, or intraventricular bleed; subarachnoid hemorrhage; subdural hemorrhage; epidural hemorrhage; extradural/extra-axial blood), diffuse injuries (punctuate hemorrhage or petecchia; swelling/edema/effacement of sulci/attenuation of gray-white matter; abnormal/compressed/displaced/asymmetric ventricles; abnormal/obliterated/hyperintense cisterns; midline shift; brain herniation; mass effect), and skull fractures (linear or depressed skull fracture in frontal, parietal, occipital or temporal bone; basilar skull fracture). Two measures were extracted from these ratings: CT-focal injury (maximum points $=6$ ) and CT- 
diffuse injury (maximum points $=7$ ). Because of the lack of normality in accuracy and RT data, Spearman correlations were computed between CT measures, lowest GCS scores, and accuracy and RT on the key ToM (switched, unwitnessed) trials.

\section{RESULTS}

\section{Task Performance}

Figure 2 displays the mean percentage accuracy for the three groups across the various combinations of switched/unswitched and witnessed/unwitnessed trial types. The repeatedmeasures analysis revealed a significant main effect for group, reflecting overall higher accuracy for children with OI than for those with TBI ( $82.74 \%$ for OI, $78.63 \%$ for Mild/ Moderate TBI, $69.16 \%$ for Severe TBI), $F(2,137)=8.08, p<.001$. Accuracy was also significantly higher for unswitched than for switched trials $(85.31 \%$ vs. $68.37 \%), F(1,137)=$ $89.45, p<.001$, and for witnessed than for unwitnessed trials ( $84.91 \%$ vs. $68.77 \%)$, $F(1,137)=89.26, p<.001$. The interaction of switched/unswitched and witnessed/ unwitnessed trials was also significant, because accuracy was lowest specifically on switched/unwitnessed trials, which are those that depend on ToM, but did not differ on the other three trial type combinations (53.92\% for switched/unwitnessed vs. 82.83-86.97\% for the other combinations), $F(1,137)=54.50, p<.001$. The latter finding indicates that the task manipulation was successful; notably, the overall accuracy rate for the switched/unwitnessed trials is only slightly higher than chance.

Most important for the current study, the three-way interaction of group membership with switched/unswitched and witnessed/unwitnessed trial types was significant, $F(2,137)=3.03$, $p=.052$. Compared to children with OI, children with severe TBI showed a larger decline in accuracy on the switched/unwitnessed trials as compared to switched/witnessed trials, $p<$. 05, suggesting a specific deficit in ToM among children with severe TBI. Indeed, their accuracy on the switched/unwitnessed trials fell below chance levels. The OI and Mild/ Moderate TBI groups did not show a significant differential decline in accuracy across those two conditions, and their accuracy on the switched/unwitnessed trials was above chance levels. Notably, the OI and TBI groups showed small differences in accuracy between the unwitnessed versus witnessed conditions for unswitched trials.

The analysis of the RT data revealed longer average RT on switched than unswitched trials, $F(1,115)=11.17, p<.001$, and unwitnessed than witnessed trials, $F(1,115)=6.56, p<.05$. However, the interaction of switched/unswitched and witnessed/unwitnessed trials was not significant, $F(1,115)=0.64, p>.10$. Moreover, the groups did not differ in average RT, and group membership did not interact with any of the trial types in predicting RT.

\section{Age At Injury and Age at Test Effects}

Our study design is cross-sectional and we imposed a relatively restricted time interval between injury and test to ensure all children were well into the chronic state of recovery from TBI. This means that age at injury is highly correlated with age at test, and one cannot discuss one without the other, or attribute effects to one rather than to the other. The two time variables are highly correlated with each other $\left(r_{\mathrm{s}}=.806 ; p<.001\right)$. Within the OI group, both age at injury and testing were correlated with accuracy on the overall task $\left(r_{s}=\right.$. $440 ; p<.001 ; r_{S}=.603 ; p<.001$, respectively), on ToM trials $\left(r_{S}=.507 ; p<.001 ; r_{S}=.624\right.$; $p<.001)$, and on non-ToM trials $\left(r_{S}=.268 ; p=.037 ; r_{S}=.429 ; p=.001\right)$. Likewise in the Mild/Moderate TBI group, age at injury and testing were correlated with accuracy on the overall task $\left(r_{S}=.411 ; p=.002 ; r_{S}=.542 ; p<.001\right)$, on only ToM trials $\left(r_{S}=.322 ; p=.015\right.$; $\left.r_{S}=.366 ; p=.006\right)$, and on non-ToM trials $\left(r_{S}=.317 ; p=.017 ; r_{S}=.498 ; p<.001\right)$.

However, in the Severe TBI group, age at injury was not correlated with accuracy on the 
overall task $\left(r_{S}=.339 ; p=.113\right)$, on ToM trials $\left(r_{S}=.256 ; p=.239\right)$, or on non-ToM trials $\left(r_{S}=.210 ; p=.336\right)$ while age at testing was correlated with overall accuracy $\left(r_{S}=.454 ; p\right.$ $=.030)$ and marginally with accuracy on non-ToM trials $\left(r_{S}=.395 ; p=.062\right)$, but not with accuracy on ToM trials $\left(r_{S}=.349 ; p=.103\right)$.

\section{Performance Monitoring}

The analysis of performance monitoring revealed a significant main effect for group, reflecting higher accuracy among children with OI as compared to TBI, as already reported. Accuracy was also significantly higher on trials following errors than on trials following correct responses $(80.80 \%$ vs. $77.11 \%), F(1,125)=12.11, p<.001$, suggesting that children monitored their performance and responded to errors with increased vigilance. However, the interaction of group membership and trial type (after error $v s$. after correct response) was not significant, $F(2,125)=0.66, p>.10$, suggesting that children with TBI did not differ from those with $\mathrm{OI}$ in their performance monitoring, despite their overall lower accuracy on the task.

The analysis of the RT data revealed marginally slower average reaction times after errors as opposed to correct responses, $F(1,123)=2.91, p<.09$. However, the groups did not differ in this respect; the interaction of group and trial type (after error $v s$. after correct response) was not significant, $F(2,123)=1.10, p>.10$.

\section{Injury Severity}

Neither CT-focal injury nor CT-diffuse injury scores was correlated with accuracy or RT on ToM trials, nor was lowest GCS score correlated with accuracy or RT on ToM trials. Furthermore, no correlation was found between CT-total injury scores and lowest GCS scores $\left(r_{s}=.034 ; p=.773\right)$.

\section{DISCUSSION}

In this study, we examined ToM in children with TBI, compared to age- and gendermatched peers with OI, using a new experimental paradigm, the Jack and Jill task. The task isolated trials that required intentional processing from those that did not, while maintaining comparable metacognitive, attention, and working memory demands. Switched, unwitnessed (ToM) trials required intentional processing, while the other trial types did not.

All children could perform the task. Although the groups differed in task accuracy, they did not differ in RT for correct responses. The fact that groups took a similar length of time to make a correct response is informative and suggests that both TBI groups attended to the task and attempted to solve it in the same time frame as did their age peers with OI.

Overall task accuracy is sensitive to the presence of TBI. More centrally, children with TBI showed a specific deficit on ToM trials, suggesting that TBI produces selectively poorer processing of trials in which inferences must be made about someone else's mental states, an effect that is magnified with increasing TBI severity. ToM trials engage a different type of processing from the non-ToM trials because even children with severe TBI were able to perform the same items correctly when they did not entail intentional demands. Intact performance on non-ToM trials suggests that children with TBI are able to keep information in mind and process that information across sequential frames. Of interest, the children with severe TBI performed more poorly than chance, which shows that they were not inattentive or random in their approach to the task but were failing to take account of what Jill must know if she did not witness the ball switch, which suggests a profound problem with intentional thinking. 
Children with TBI have working memory challenges (e.g., Roncadin, Guger, Archibald, Barnes, \& Dennis, 2004). Like any cognitive task, ToM tasks draw on general purpose functions, such as working memory and cognitive inhibition during typical development (Astington \& Jenkins, 1999; Carlson, Moses, \& Claxton, 2004; Gordon \& Olson, 1998; Moses, 2001) and after TBI (Dennis, Agostino, Roncadin, \& Levin, 2009); however, working memory does not explain the pattern or group differences (groups performed similarly on non-ToM items) or the fact that ToM items were more difficult for all groups, especially those with TBI. The performance on ToM trials suggests a specific problem with intentional processing, and is not secondary to problems in other general cognitive processes.

Because the trials in the Jack and Jill task were randomly intermixed, not blocked, participants were required to flexibly engage and disengage ToM processes throughout the task, not simply to apply the same ToM process to every trial. This property of the task allowed us to explore two temporal features of performance: the ability to make performance adjustments, and the consequences of post-trial adjustments on subsequent performance. All groups responded to errors with increased performance monitoring so they were less likely to make an error on the next trial. Taken together, the RT and performance monitoring data show that all three groups not only attended to the task, but also monitored their own task errors so as to sustain and optimize their individual performances throughout the task. It is, therefore, unlikely that group differences can be explained on the basis of attentional or motivational limitations. In assessing the ecological implication of deficits on ToM tasks, both performance accuracy and performance monitoring should be measured, and their lack of congruence may be informative of mechanisms underlying cognitive deficits.

Debate continues about whether and how CT reports might better predict functional outcome than GCS scores alone (Levin et al., 2008; Yuh, Cooper, Ferguson, \& Manley, 2012). In addition to group comparisons, we considered within-group analyses for the TBI groups; specifically, whether ToM performance varied in relationship to injury severity, and whether coding by GCS score (the basis of group assignment) produced a different pattern of results from ratings of day-of-injury CT scan reports. With respect to ToM outcome, dayof-injury CT scan reports did not add to discriminability among individuals with TBI in the moderate to severe range. Of interest, the correlation between the GCS score and CT findings was trivial, suggesting that these may capture different dimensions of TBI severity. One reason for the absence of associations between day-of-injury CT findings and performance on the Jack and Jill task is that our ratings did not consider injury location, which, along with other characteristics of damage such as hemosiderin deposits and atrophy, is likely better assessed by MRI. Day-of-injury CT scans monitor a series of insults to the brain, the evolution of which can only be judged at a later time point (Wu et al., 2010). CT information might not be predictive of later MRI status, which may be more clearly related to social outcomes involving ToM.

Two limitations of our study should be noted. We have studied one form of cognitive ToM, not the broad constellations of functions now included under the ToM label. Group differences in SCI were no longer significant when injury mechanism was taken into account, which highlights the close relation between these two variables. While future studies might compare TBI groups differing independently in SCI and injury mechanism, the fact that we did not so is a limitation of our study and means that we cannot evaluate the relative contributions of SCI and injury mechanism to our results.

Childhood TBI is associated with de novo changes in personality and behavior (Dennis, Guger, Roncadin, Barnes, \& Schachar, 2001a, 2001b; Levin et al., 2004, 2007; Max et al., 
$2005,2006,2011)$. The social outcomes of children with TBI are often suboptimal, and include difficulties in social problem solving, peer group interactions, and social cognition. Many of these domains involve cognitive ToM as part of a constellation of social skills. In this study, we have isolated cognitive ToM from the task in which it is instantiated and shown that children with TBI have relatively more difficulty with ToM than with items of similar form and, furthermore, that the deficit is sensitive to TBI severity.

Although cognitive ToM is only one component of social function, it is an important one. The ability to engage and disengage ToM thinking, to be able to read others' minds when it is appropriate to do so, is an important component of flexible social interactions. Deficits in this area, of themselves and in concert with other impairments in affective ToM and social problem solving, limit the child's social thinking and the kinds of social interactions in which they can participate. More broadly, ToM deficits are a component of the child's success in the social world.

This study documents the sensitivity of a novel ToM task (the Jack and Jill task) to impairments in ToM in children with TBI. As group differences emerged on intentional (ToM) but not non-intentional trial items, the findings suggest that school-age children and adolescents with TBI have specific deficits in this social skills domain relative to peers with OI. We also found a dissociation between level of performance and error monitoring. Because all groups, regardless of their level of performance, responded to errors by improving performance on post-error trials, the findings demonstrate a level of performance monitoring in a social cognitive context in children with TBI. The brain bases of the various ToM deficits in childhood TBI remain to be established.

\section{Acknowledgments}

Preparation of this study was supported by National Institute of Neurological Diseases and Stroke Grant 1 RO1 HD 04946, "Social Outcomes in Pediatric Traumatic Brain Injury."

\section{References}

Astington, JW. Sometimes necessary, never sufficient: False-belief understanding and social competence. In: Repacholi, B.; Slaughter, V., editors. Individual differences in theory of mind: Implications for typical and atypical development. New York: Psychology Press; 2003.

Astington JW, Jenkins JM. A longitudinal study of the relation between language and theory-of-mind development. Developmental Psychology. 1999; 35(5):1311-1320. [PubMed: 10493656]

Bartsch K, Wellman H. Young children's attribution of action to beliefs and desires. Child Development. 1989; 60(4):946-964. [PubMed: 2758888]

Bibby H, McDonald S. Theory of mind after traumatic brain injury. Neuropsychologia. 2005; 43(1): 99-114. [PubMed: 15488910]

Brown RL. Epidemiology of injury and the impact of health disparities. Current Opinion in Pediatrics. 2010; 22:321-325. [PubMed: 20375897]

Carlson SM, Moses LJ, Claxton LJ. Individual differences in executive functioning and theory of mind: An investigation of inhibitory control and planning ability. Journal of Experimental Child Psychology. 2004; 87(4):299-319. [PubMed: 15050456]

Chapman SB, Sparks G, Levin HS, Dennis M, Roncadin C, Zhang L, Song J. Discourse macrolevel processing after severe pediatric traumatic brain injury. Developmental Neuropsychology. 2004; 25:37-60. [PubMed: 14984328]

Chevrier AD, Noseworthy MD, Schachar R. Dissociation of response inhibition and performance monitoring in the stop signal task using event-related fMRI. Human Brain Mapping. 2007; 28:1347-1358. [PubMed: 17274022] 
Dennis M, Agostino A, Roncadin C, Levin H. Theory of mind depends on domain-general executive functions of working memory and cognitive inhibition in children with traumatic brain injury. Journal of Clinical and Experimental Neuropsychology. 2009; 13:835-847. [PubMed: 19221924]

Dennis M, Barnes M. Oral discourse skills in children and adolescents after early-onset hydrocephalus: Linguistic ambiguity, figurative language, speech acts, and script-based inferences. Journal of Pediatric Psychology. 1993; 18:639-652. [PubMed: 8295084]

Dennis M, Barnes MA. Speech acts after mild or severe childhood head injury. Aphasiology. 2000; 14(4):391-405.

Dennis M, Barnes MA. Comparison of literal, inferential, and intentional text comprehension in children with mild or severe closed head injury. Journal of Head Trauma Rehabilitation. 2001; 16:1-14. [PubMed: 11277847]

Dennis M, Barnes MA, Donnelly RE, Wilkinson M, Humphreys RP. Appraising and managing knowledge: Metacognitive skills after childhood head injury. Developmental Neuropsychology. 1996; 12:77-103.

Dennis M, Francis DJ, Cirino PT, Schachar R, Barnes MA, Fletcher JM. Why IQ is not a covariate in cognitive studies of neurodevelopmental disorders. Journal of the International Neuropsychological Society. 2009; 15:331-343. [PubMed: 19402919]

Dennis M, Guger S, Roncadin C, Barnes M, Schachar R. Attentional-inhibitory control and socialbehavioral regulation after childhood closed head injury. Do biological developmental and recovery variables predict outcome? Journal of the International Neuropsychological Society. 2001a; 7:683-692. [PubMed: 11575590]

Dennis M, Guger S, Roncadin C, Barnes M, Schachar R. Attentional control and social discourse after childhood closed head injury: Are frontal contusions, age at injury and time since injury predictive? Brain and Cognition. 2001b; 48:197-200.

Dennis M, Purvis K, Barnes MA, Wilkinson M, Winner E. Understanding of literal truth, ironic criticism, and deceptive praise after childhood head injury. Brain and Language. 2001; 78:1-16. [PubMed: 11412012]

Dennis M, Wilkinson M, Humphreys RP. How children with head injury represent real and deceptive emotion in short narratives. Brain and Language. 1998; 61:450-483. [PubMed: 9570873]

Filippova E, Astington JW. Further development in social reasoning revealed in discourse irony understanding. Child Development. 2008; 79(1):126-138. [PubMed: 18269513]

Frith U, Frith C. The biological basis of social interaction. Current Directions in Psychological Science. 2003; 10:151-155.

Gordon ACL, Olson DR. The relation between acquisition of a theory of mind and the capacity to hold in mind. Journal of Experimental Child Psychology. 1998; 68(1):70-83. [PubMed: 9473316]

Greenbaum RL, Stevens SA, Nash K, Koren G, Rovet J. Social cognitive and emotion processing abilities of children with fetal alcohol spectrum disorders: A comparison with attention deficit hyperactivity disorder. Alcoholism, Clinical and Experimental Research. 2009; 33(10):1656-1670.

Hanten G, Dennis M, Zhang L, Barnes M, Roberson G, Archibald J, Levin HS. Childhood head injury and metacognitive processes in language and memory. Developmental Neuropsychology. 2004; 25(1-2):85-106. [PubMed: 14984330]

Hein G, Singer T. I feel how you feel but not always: the empathic brain and its modulation. Current Opinion in Neurobiology. 2008; 18:153-158. [PubMed: 18692571]

Hein G, Silani G, Preuschoff K, Batson CD, Singer T. Neural responses to ingroup and outgroup members' suffering predict individual differences in costly helping. Neuron. 2010; 68:149-160. [PubMed: 20920798]

Hill EL, Frith U. Understanding autism: Insights from mind and brain. Philosophical Transactions of the Royal Society of London Series B, Biological Sciences. 2003; 358:281-289.

Howard I, Joseph JG, Natale JE. Pediatric traumatic brain injury: Do racial/ethnic disparities exist in brain injury severity, mortality, or medical disposition? Ethnicity \& Disease. 2005; 15(Suppl 5): 51-56.

Langlois JA, Rutland-Brown W, Thomas KE. The incidence of traumatic brain injury among children in the United States: Differences by race. Journal of Head Trauma Rehabilitation. 2005; 20:229238. [PubMed: 15908823] 
Levin H, Hanten G, Max J, Li X, Swank P, Ewing-Cobbs L, Schachar R. Symptoms of attentiondeficit/hyperactivity disorder following traumatic brain injury in children. Journal of Developmental and Behavioral Pediatrics. 2007; 28(2):108-118. [PubMed: 17435461]

Levin HS, Hanten G, Roberson G, Li X, Ewing-Cobbs L, Dennis M, Swank P. Prediction of cognitive sequelae based on abnormal computed tomography findings in children following mild traumatic brain injury. Journal of Neurosurgery Pediatrics. 2008; 1(6):461-470. [PubMed: 18518697]

Levin HS, Zhang L, Dennis M, Ewing-Cobbs L, Schachar R, Max J, Hunter JV. Psychosocial outcome of TBI in children with unilateral frontal lesions. Journal of the International Neuropsychological Society. 2004; 10:305-316. [PubMed: 15147589]

Martín-Rodríguez JF, León-Carrión J. Theory of mind deficits in patients with acquired brain injury: A quantitative review. Neuropsychologia. 2010; 48(5):1181-1191. [PubMed: 20153762]

Max JE, Keatley E, Wilde EA, Bigler ED, Levin HS, Schachar RJ, Yang TT. Anxiety disorders in children and adolescents in the first six months after traumatic brain injury. The Journal of Neuropsychiatry and Clinical Neurosciences. 2011; 23(1):29-39. [PubMed: 21304136]

Max JE, Levin HS, Landis J, Schachar R, Saunders AE, Ewing-Cobbs L, Dennis M. Predictors of personality change due to traumatic brain injury in children and adolescents in the first six months after injury. Journal of the American Academy of Child and Adolescent Psychiatry. 2005; 44:435442 .

Max JE, Levin HS, Schachar R, Landis J, Saunders AE, Ewing-Cobbs L, Dennis M. Predictors of personality change due to traumatic brain injury in children and adolescents 6 to 24 months after injury. Journal of Neuropsychiatry and Clinical Neurosciences. 2006; 18:21-32. [PubMed: 16525067]

McKinlay A, Kyonka EGE, Grace RC, Horwood LJ, Fergusson DM, MacFarlane MR. An investigation of the pre-injury risk factors associated with children who experience traumatic brain injury. Injury Prevention. 2010; 16:31-35. [PubMed: 20179033]

Meltzoff AN. What infant memory tells us about infantile amnesia: Long-term recall and deferred imitation. Journal of Experimental Child Psychology. 1995; 59(3):497-515. [PubMed: 7622990]

Meltzoff, AN.; Gopnik, A.; Repacholi, B. Toddlers understanding of intentions, desires and emotions: Explorations of the dark ages. In: Zelazo, P., editor. Developing theories of intention. New Jersey: Erlbaum; 1999.

Moses LJ. Executive accounts of theory of mind development. Child Development. 2001; 72:688-690. [PubMed: 11405573]

Muscara F, Catroppa C, Eren S, Anderson V. The impact of injury severity on long-term social outcome following paediatric traumatic brain injury. Neuropsychological Rehabilitation. 2009; 19(4):541-546. [PubMed: 18839384]

Olsson A, Nearing KL, Phelps EA. Learning fears by observing others: The neural systems of social fear transmission. Social Cognitive and Affective Neuroscience. 2007; 2:3-11. [PubMed: 18985115]

Ornstein TJ, Levin HS, Chen S, Hanten G, Ewing-Cobbs L, Dennis M, Schachar R. Performance monitoring in children following traumatic brain injury. Journal of Child Psychology and Psychiatry. 2009; 50(4):506-513. [PubMed: 19207625]

Parslow RC, Morris KP, Tasker RC, Forsyth RJ, Hawley CA. Epidemiology of traumatic brain injury in children receiving intensive care in the UK. Archives of Disease in Childhood. 2005; 90:11821187. [PubMed: 16049060]

Premack D, Woodruff G. Chimpanzee problem-solving: A test for comprehension. Science. 1978; 202:532-535. [PubMed: 705342]

Robinson EJ. Six-year-olds' contradictory judgments about knowledge and beliefs. Trends in Cognitive Sciences. 2003; 7:235-237. [PubMed: 12804686]

Roncadin C, Guger S, Archibald J, Barnes M, Dennis M. Working memory after mild, moderate, or severe childhood head injury. Developmental Neuropsychology. 2004; 25:21-36. [PubMed: 14984327]

Shamay-Tsoory SG, Aharon-Peretz J. Dissociable prefrontal networks for cognitive and affective theory of mind: A lesion study. Neuropsychologia. 2007; 45(13):3054-3067. [PubMed: 17640690] 
Shammi P, Stuss DT. Humor appreciation. A role for the right frontal lobe. Brain. 1999; 122:657-666. [PubMed: 10219779]

Singer T, Seymour B, O’Doherty J, Kaube H, Dolan RJ, Firth CD. Empathy for pain involves the affective but not sensory components of pain. Science. 2004; 303:1157-1162. [PubMed: 14976305]

Sodian B, Taylor C, Harris PL, Perner J. Early deception and the child's theory of mind: False trails and genuine markers. Child Development. 1991; 62:468-483.

Stuss DT, Gallup GG Jr, Alexander MP. The frontal lobes are necessary for 'theory of mind'. Brain. 2001; 124(Pt 2):279-286. [PubMed: 11157555]

Teasdale G, Jennett B. Assessment of coma and impaired consciousness: A practical scale. Lancet. 1974; 2(7872):81-84. [PubMed: 4136544]

Uekermann J, Kraemer M, Abdel-Hamid M, Schimmelmann BG, Hebebrand J, Daum I, Kis B. Social cognition in attention-deficit hyperactivity disorder (ADHD). Neuroscience and Biobehavioral Reviews. 2010; 34(5):734-743. [PubMed: 19857516]

Wellman HM, Cross D, Watson J. Meta-analysis of theory-of-mind development: The truth about false belief. Child Development. 2001; 72(3):655-684. [PubMed: 11405571]

Wimmer H, Perner J. Beliefs about beliefs: Representation and constraining function of wrong beliefs in young children's understanding of deception. Cognition. 1983; 13(1):103-128. [PubMed: 6681741]

Wobbrock, JO.; Findlater, L.; Gergle, D.; Higgins, JJ. User-defined gestures for surface computing. Proceedings of the ACM Conference on Human Factors in Computing Systems '11: The Aligned Rank Transform for nonparametric factorial analyses using only ANOVA procedures; Vancouver, British Columbia. New York: ACM Press; 2011. p. 143-146.

Wu TC, Wilde EA, Bigler ED, Li X, Merkley TL, Yallampalli R, Levin HS. Longitudinal changes in the corpus callosum following pediatric traumatic brain injury. Developmental Neuroscience. 2010; 32(5-6):361-373. [PubMed: 20948181]

Yeates K, Bigler E, Dennis M, Gerhardt C, Rubin K, Stancin T, Vannatta K. Social outcomes in childhood brain disorder: A heuristic integration of social neuroscience and developmental psychology. Psychological Bulletin. 2007; 133(3):535-556. [PubMed: 17469991]

Yeates KO, Taylor HG. Predicting premorbid neuropsychological functioning following pediatric traumatic brain injury. Journal of Clinical and Experimental Neuropsychology. 1997; 19:825-837. [PubMed: 9524877]

Yates PJ, Williams WH, Harris A, Round A, Jenkins R. An epidemiological study of head injuries in a UK population attending an emergency department. Journal of Neurology, Neurosurgery, \& Psychiatry. 2006; 77:699-701.

Yuh EL, Cooper SR, Ferguson AR, Manley GT. Quantitative CT improves outcome prediction in acute traumatic brain injury. Journal of Neurotrauma. 2012; 29:735-746. [PubMed: 21970562] 

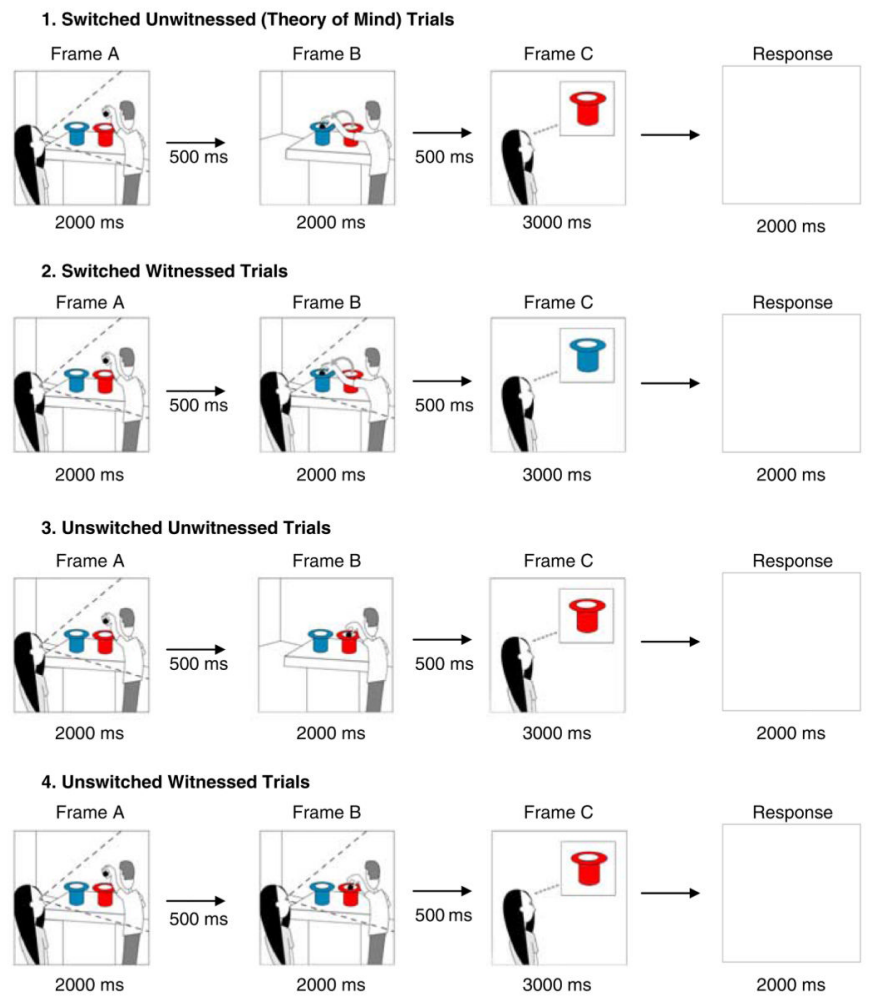

Fig. 1.

Sample stimuli for Jack and Jill task illustrating the four trial types. In these examples, the correct response is "yes." 

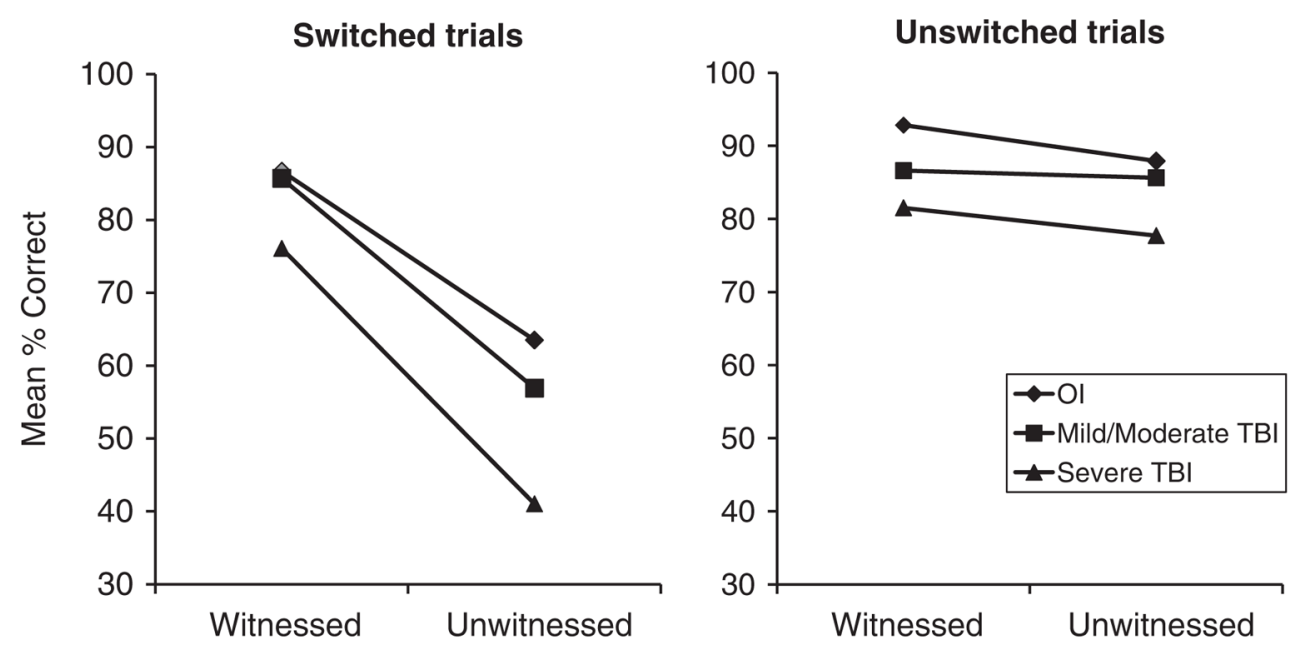

Fig. 2.

Accuracy results for the four trial types. Groups differed significantly in overall accuracy, with the OI group having a significantly higher proportion of correct responses. Accuracy was lowest on switched/unwitnessed trials, which are those that depend on Theory of Mind (ToM), with the Severe TBI (traumatic brain injury) group showing the largest decline in accuracy on these trials such that their performance was below chance levels. 


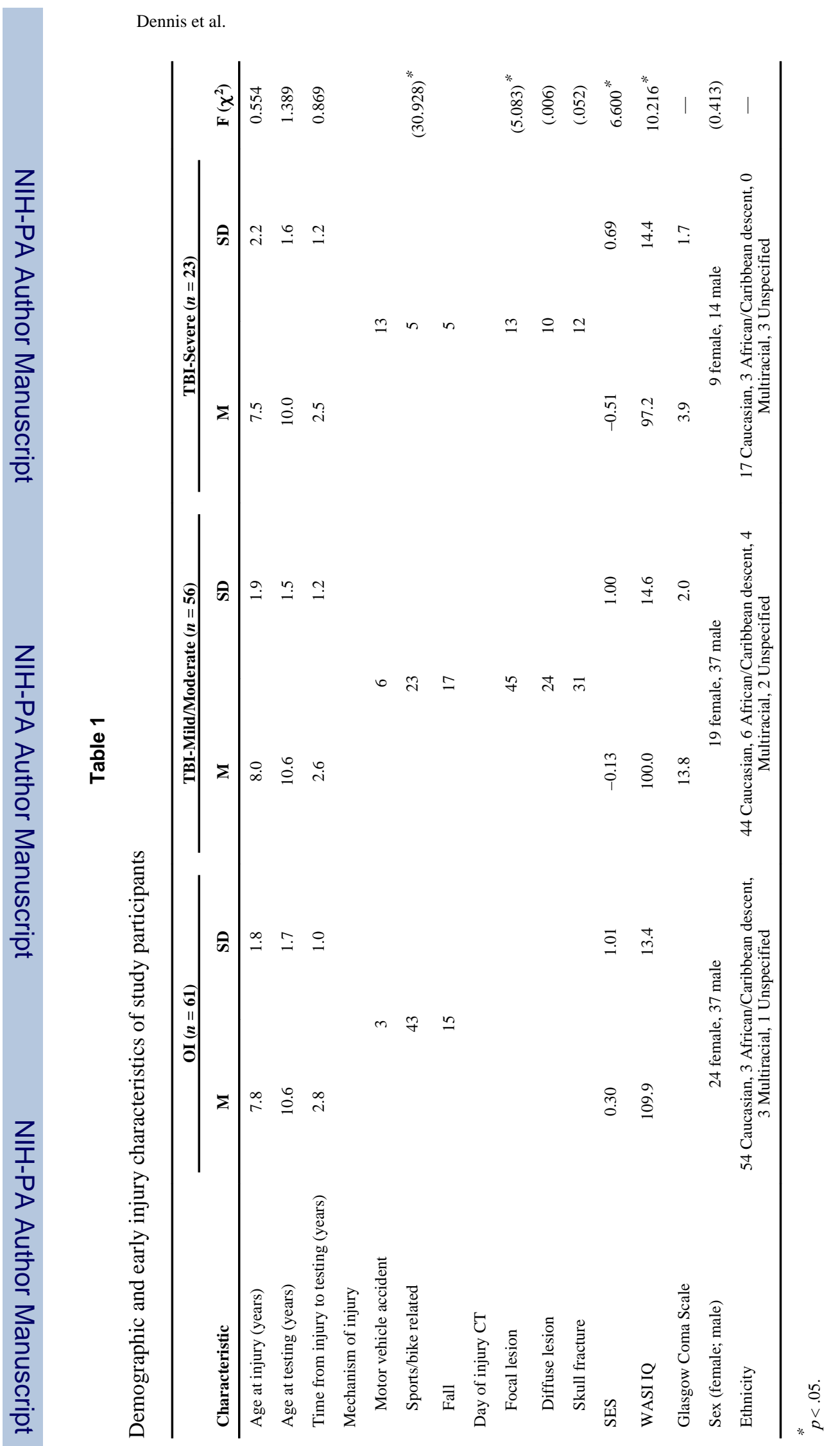

J Int Neuropsychol Soc. Author manuscript; available in PMC 2013 September 01. 